\title{
Développement de Cyanobactéries toxiques dans l'Ouest de la France. Détection de la toxicité par des tests sur Artemia salina
}

\author{
C. Vezie \\ K. Sivonen ${ }^{2}$ \\ L. Brient ${ }^{1}$ \\ G. Bertru1 \\ J.-C. Lefeuvre'
}

Mots clés : Cyanobactéries, toxines, détection, bioessai, test souris, Artemia salina

Une recherche de toxicité a été réalisée sur dix-huit souches isolées à partir d'efflorescences de cyanobactéries récoltées dans l'Ouest de la France et deux souches provenant de cultures de collection. Un biotest basé sur la sensibilité de larves d'une crevette marine Artemia salina a été développé et comparé à des bioessais sur souris, outil de référence pour la détection des toxines de cyanobactéries.

Parmi les souches provenant du milieu naturel, six se sont avérées hépatotoxiques par les bioessais sur souris. Pour l'hépatotoxicité, les réponses du test $A$. salina recouvrent celles données par le test souris.

\section{Development of toxic cyanobacteria in western France. Detection of toxicity with Artemia salina tests}

Keywords : Cyanobacteria, toxin, detection, bioassay, mouse test, Artemia salina

Toxicity of eighteen strains isolated from cyanobacterial blooms collected in western France and two strains from culture collection was investigated. A biotest based on sensitivity of brine shrimp Artemia salina larvae was developed and compared to mouse bioassay, the reference test for detection of cyanobacterial toxins. From the strains collected on the field, six were hepatotoxic to mice. A good similarity between $A$. salina test and mouse test was shown.

\section{Introduction}

Aux symptômes habituels de l'eutrophisation s'ajoutent les productions éventuelles de toxines par les efflorescences à cyanobactéries dont 40 à $75 \%$ sont susceptibles de produire soit des hépatotoxines, soit des neurotoxines. Les genres Microcystis, Nodularia, Oscillatoria synthétisent les premières alors que les secondes le sont par Aphanizomenon, Anabaena, Gomphosphaeria (Carmichael \& Gorham 1978, Codd \&

1. Laboratoire d'Evolution des systèmes Naturels et Modifiés, Université de Rennes 1, Av. du Général Leclerc, 35042 Rennes Cedex, France.

2. Department of Applied Chemistry and Microbiology. P.O. BOX 27, SF-00014, University of Helsinki.

Article available at http://www.limnology-journal.org or http://dx.doi.org/10.1051/limn/1996009
Poon 1988, Watanabe et al. 1991). Des hépatotoxicités, des dermatotoxcités ainsi que des syndromes gastro-intestinaux ont été rapportés à des efflorescences à cyanobactéries chez l'homme (Skulberg et al.1984, Carmichael \& Falconer 1993) et l'animal (Beasley et al. 1989).

Alors que ces problèmes ont été observés et décrits en Amérique du Nord, en Australie, en Scandinavie et dans plusieurs pays européens, il ne semble pas avoir reçu en France l'attention qu'ils méritaient. L'eutrophisation accélérée des divers systèmes aquatiques et la forte dépendance de la Bretagne vis-à-vis des eaux de surface pour son alimentation en eau potable font qu'il devenait urgent de dresser un premier bilan vis-àvis des cyanobactéries toxinogènes. Pour ce faire, nous avons souhaité nous assurer le concours de K. Sivonen (University of Helsinki) pour l'identification des di- 
verses toxines et l'utilisation du test souris. Si ce test, réalisé par injection intrapéritonale, constitue la méthode de référence pour caractériser les toxines et les symptômes de celles-ci (Sivonen et al. 1990), il n'en demeure pas moins assez contraignant dans sa réalisation régulière. Aussi, avons-nous saisi l'occasion de développer en parallèle un bioessai basé sur l'utilisation de larves d'Artemia salina qui, sans se substituer au test souris, pourrait donner des informations utiles quant à la présence des toxines.

\section{Matériels et méthodes}

\subsection{Isolement et culture des souches cyanobacté- riennes}

A partir d'efflorescences récoltées de 1991 à 1993 (Fig. 1 et Tableau 1), 18 souches ont été isolées par la méthode de Shirai et al. (1989). Après purification, les algues cultivées en milieu de Jaworski sont lyophilisées et conservées à $-20^{\circ} \mathrm{C}$. Deux souches non toxiques, Microcystis aeruginosa Kütz. CCAP 1450/1 et Aphanizomenon flos-aquae Ralfs CCAP1401/4, proviennent de «Culture Collection of Algae and Protozoa» (U.K.).

\subsection{Extraction des toxines}

Les cellules lyophilisées sont mises en suspension dans de l'acide acétique $5 \%(10 \mathrm{mg} / \mathrm{ml})$. La dispersion et la rupture des cellules sont réalisées par deux passages de 15 secondes à la sonde à ultrasons $(100 \mathrm{~W}, 19$ $\mathrm{mm}$ ) à 30 minutes d'intervalle. La suspension, filtrée sur Whatman GFC, est lavée avec la solution d'extraction, puis éluée sur cartouche Bond Elut C18, celle-ci étant lavée avec de l'eau déionisée et du méthanol 20 $\%$ (Luukkainen et al. 1993). Les toxines retenues sur la cartouche sont récupérées avec du méthanol pur. L'extrait est conservé à $4^{\circ} \mathrm{C}$.
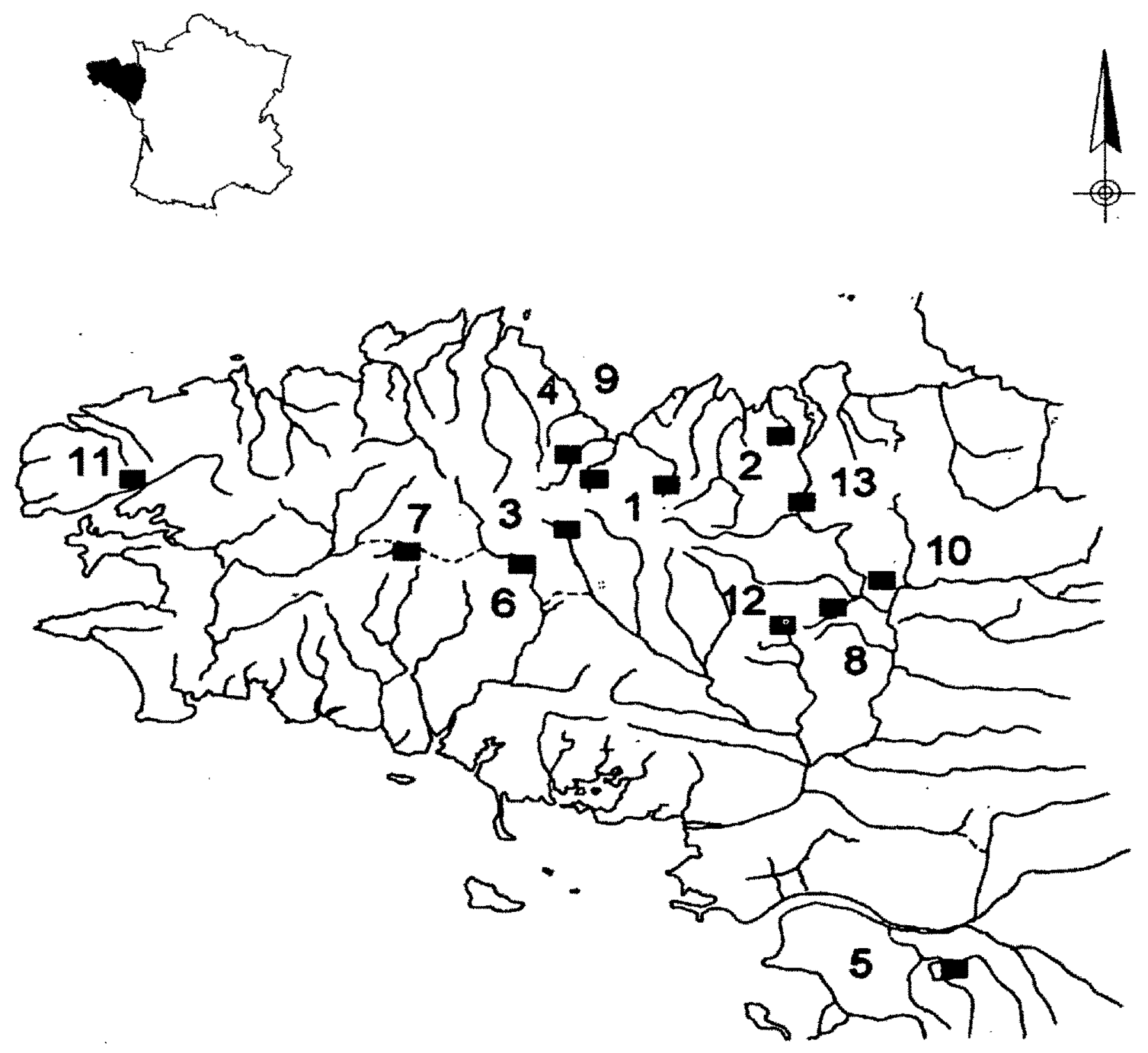

Fig. 1. Sites de prélèvements des efflorescences à cyanobactéries.

Fig. 1. Location of sites where samples of cyanobacterial blooms were collected. 
Tableau 1. Liste des souches isolees a partir du milieu naturel (*: cf carte Fig. 1)

Table 1. List of natural strains isolated (* cf. map, Fig. 1).

\begin{tabular}{|c|c|c|c|}
\hline Espèces & Sites d'chantillonnage* & Dates & Codes \\
\hline Microcystis aeruginosa & $\begin{array}{l}\text { 1- Retenue de l'Arguenon (22) } \\
\text { 2- Etang de Bobital (22) } \\
3 \text { - Etang de Bosméléac (22) } \\
\text { 4- Retenue du Gouët (22) } \\
5 \text { - Lac de Grand-Lieu (44) } \\
\text { 6- Retenue de Guerlédan (56) } \\
7 \text { - Etang de Glomel (22) } \\
8 \text { - Retenue de la Cheze au Pont-Muzard (35) } \\
\text { 9- Retenue de l'Urne }\end{array}$ & $\begin{array}{l}21 / 09 / 92 \\
01 / 09 / 93 \\
03 / 09 / 91 \\
03 / 06 / 91 \\
03 / 05 / 91 \\
04 / 09 / 91 \\
04 / 09 / 91 \\
21 / 10 / 91 \\
28 / 08 / 92\end{array}$ & $\begin{array}{l}\text { MA92 } \\
\text { MBL93 } \\
\text { MBC91 } \\
\text { MG91 } \\
\text { MGL91 } \\
\text { MGR91 } \\
\text { MMC91 } \\
\text { MPM91 } \\
\text { MU921 }\end{array}$ \\
\hline Aphanizomenon flos-aquae & $\begin{array}{l}4 \text { - Retenue du Gouët (22) } \\
10 \text { - Etang de l'INRA du Rheu (35) } \\
8 \text { - Retenue de la Cheze au Pont-Muzard (35) } \\
13 \text { - Retenue de Rophémel (22) }\end{array}$ & $\begin{array}{r}03 / 06 / 91 \\
06 / 91 \\
21 / 10 / 91 \\
01 / 09 / 93\end{array}$ & $\begin{array}{l}\text { ApG91 } \\
\text { ApI91 } \\
\text { ApPM91 } \\
\text { ApR93 }\end{array}$ \\
\hline Anabaena circinalisna & 5- Lac de Grand-Lieu (44) & $31 / 05 / 91$ & AnGL91 \\
\hline Oscillatoria sp. & $\begin{array}{l}11 \text { - Retenue de Goarem Vorz (29) } \\
12 \text { - Etang des Forges (56) } \\
5 \text { - Lac de Grand-Lieu (44) } \\
6 \text { - Retenue de Guerlédan (56) }\end{array}$ & $\begin{array}{r}06 / 91 \\
01 / 09 / 93 \\
31 / 05 / 91 \\
04 / 09 / 91\end{array}$ & $\begin{array}{l}\text { OBT91 } \\
\text { OF93 } \\
\text { OGL91 } \\
\text { OGR91 }\end{array}$ \\
\hline
\end{tabular}

\subsection{Bioessais sur souris}

$1 \cdot \mathrm{ml}$ d'une suspension de cellules lyophilisées dans de l'eau distillée ( $50 \mathrm{mg}$ de poids $\mathrm{sec} / \mathrm{ml}$ ) est injecté à des souris femelles NIH/S de 20 à $25 \mathrm{~g}$ provenant de l'Université d'Helsinki. Les symptômes présentés par les souris sont observés pendant quatre heures. Audelà de ce délai, la mort n'est pas attribuée aux toxines cyanobactériennes (Gorham 1964). Après dissection, le foie est observé pour vérifier la présence d'hépatotoxines.

\subsection{Bioessais sur Artemia salina}

Des oeufs d'A. salina lyophilisés sont incubés dans de l'eau salée ( $40 \mathrm{~g}$ de sel marin dans un litre d'eau distillée), oxygénée, brassée et maintenue à $25^{\circ} \mathrm{C}$. L'éclosion intervient au bout de 24 à 30 heures. Les larves sont utilisées dans les 24 heures .

Après extraction de $20 \mathrm{mg}$ de cellules lyophilisées dans $20 \mathrm{ml}$ d'acide acétique et après évaporation du méthanol, l'extrait sec est suspendu dans $2 \mathrm{ml}$ d'eau salée. A partir de cette solution quatre dilutions au demi sont réalisées à l'aide de l'eau salée. Les cinq concentrations sont testées en triple exemplaire pour chaque échantillon.

Les tests sont réalisés dans des plaques nunclon constituées de 96 puits de $400 \mu \mathrm{l}$. Dans chaque puits sont introduits $50 \mu 1$ d'eau salée contenant 10 à 40 larves et $300 \mu 1$ d'échantillon (Kiviranta et al. 1991). Les plaques sont incubées à $25^{\circ} \mathrm{C}$ à la lumière. Six té- moins par plaque sont réalisés avec $300 \mu \mathrm{l}$ d'eau salée. Deux comptages des larves mortes ou ayant des mouvements anormaux sont réalisés au bout de 24 heures et de 40 heures. Après le deuxième comptage, les larves vivantes sont tuées avec de l'acide phosphorique $25 \%$ afin de compter la totalité des individus. Les résultats sont alors exprimés en pourcentage de mortalité après 24 et 40 heures de test.

\section{Résultats et discussion}

L'objectif de ce travail étant de substituer un test simple sur Artemia salina à celui plus complexe et plus coûteux habituellement utilisé, nous avons effectué une comparaison entre les résultats obtenus par l'une et l'autre méthode. Les résultats obtenus par les bioessais sur souris sont reportés dans le Tableau 2. Parmi les vingt souches étudiées, six de $M$. aeruginosa et une de Anabaena circinalis Rabenh. se sont avérées toxiques. Les souris ayant reçu une injection à partir des cinq souches de $M$. aeruginosa MA92, MBC91, MG91, MGL91, MU921 et de A. circinalis AnGL91 ont présenté des signes identiques à ceux provoqués par les hépatotoxines, faiblesse, paralysie, pâleur des extrémités (Hughes et al. 1958, Falconer et al. 1983) et sont mortes une à deux heures après l'injection. Après dissection, l'observation du foie a révélé une coloration sombre, due à une accumulation du sang dans cet organe (Theiss et.al. 1988). Seule la souche de $M$. aeruginosa MBL93 a provoqué la mort des souris 1 h 30 
Tableau 2. Résultats du bioessai sur souris (nT non toxique; T toxique; HT Hépatotoxique).

Table 2. Results of mouse bioassay ( $\mathrm{nT}$ : non toxic, $\mathrm{T}:$ toxic, HT : hepatotoxic).

\begin{tabular}{|c|c|c|c|}
\hline Espèces & Symptômes & $\begin{array}{l}\text { Temps } \\
\text { de survie }\end{array}$ & Toxicité \\
\hline \multicolumn{4}{|l|}{ M. aeruginosa } \\
\hline CCAP1450/1 & & & nT \\
\hline MA92 & convulsions, foie sombre & $1 \mathrm{~h} 25$ & HT \\
\hline MBL93 & $\begin{array}{l}\text { faiblesse, paralysie, foie } \\
\text { et extrémités normaux }\end{array}$ & $1 \mathrm{~h} 25$ & $\mathrm{~T}$ \\
\hline MBC91 & faiblesse, foie sombre & $1 \mathrm{~h} 15$ & HT \\
\hline MG91 & $\begin{array}{l}\text { faiblesse, oreilles pâles, } \\
\text { foie sombre }\end{array}$ & $1 \mathrm{~h} 25$ & HT \\
\hline MGL91 & paralysie, foie sombre & $1 \mathrm{ho0}$ & $\mathrm{HT}$ \\
\hline MGR91 & & & $\mathrm{nT}$ \\
\hline MMC91 & & & $\mathrm{nT}$ \\
\hline MPM91 & & & $\mathbf{n T}$ \\
\hline MU921 & paralysie, foie sombre & I hoo & $\mathrm{HT}$ \\
\hline \multicolumn{4}{|l|}{ A. flos-aquae } \\
\hline CCAP1401/4 & & & $\mathrm{nT}$ \\
\hline ApG91 & & & nT \\
\hline ApI91 & & & nT \\
\hline ApPM & faiblesse & $>4 h$ & $\mathrm{nT}$ \\
\hline ApR93 & faiblesse & $>4 h$ & nT \\
\hline \multicolumn{4}{|l|}{ A. circinalis } \\
\hline AnGL91 & foie sombre & $1 \mathrm{ho0}$ & HT \\
\hline \multicolumn{4}{|c|}{ Oscillatoria sp. } \\
\hline OВT91 & & & $n T$ \\
\hline OF93 & & & $\mathrm{nT}$ \\
\hline OGL91 & & & $\mathrm{nT}$ \\
\hline OGR91 & & & $\mathrm{nT}$ \\
\hline
\end{tabular}

après l'injection, avec des signes de paralysie mais sans pâleur des extrémités. De plus, après dissection, le foie était normal. Cette souche ne produit donc pas d'hépatotoxine mais un ou plusieurs autres composés toxiques pour les souris.

Malgré les signes de faiblesse provoqués, les souches d'A. flos-aquae ApPM91 et ApR93 sont considérées comme ne contenant pas de toxine car les souris sont mortes après plus de quatre heures de test.

Pour leur part, Kiviranta et al. (1991), se référant au test Artemia salina, classent comme toxiques les souches pour lesquelles la concentration de $10 \mathrm{mg} / \mathrm{ml}$ (concentration initiale de l'échantillon) provoque, au bout de 24 heures, une mortalité de plus de $50 \%$ des larves de ce crustacé et celle de $5 \mathrm{mg} / \mathrm{ml}$ entraîne la mort de plus de $20 \%$ des individus. Ces valeurs ont été choisies parce qu'elles permettaient une bonne correspondance avec les bioessais sur souris:
Tableau 3. Résultats des bioessais sur A. salina (pourcentages de mortalité) ${ }^{*}$ poids sec de l'échantillon initial ; ** nT non toxique ; T toxique.

Table 3. Results of A. salina bioassay (percentage mortality). * Initial dry weight of sample, ${ }^{* *} \mathrm{nT}:$ non toxic, $\mathrm{T}:$ toxic.

\begin{tabular}{|c|c|c|c|c|c|c|c|}
\hline \multicolumn{2}{|l|}{ Souches } & $\begin{array}{c}10 \\
\mathrm{mg} / \mathrm{ml}^{*}\end{array}$ & $\begin{array}{c}5 \\
\mathrm{mg} / \mathrm{ml}^{*}\end{array}$ & $\begin{array}{c}2,5 \\
\mathrm{mg} / \mathrm{ml} l^{*}\end{array}$ & $\begin{array}{c}1,25 \\
\mathrm{mg} / \mathrm{m} l^{*}\end{array}$ & $\begin{array}{c}0,625 \\
\mathrm{mg} / \mathrm{m} l^{*}\end{array}$ & $\begin{array}{l}\text { Toxi- } \\
\text { cité }\end{array}$ \\
\hline \multicolumn{8}{|c|}{ M. aeruginosa } \\
\hline \multirow[t]{2}{*}{ CCAP1450/1 } & $24 \mathrm{~h}$ & 10,5 & 0,7 & 0,4 & 0,6 & 0 & \\
\hline & $40 \mathrm{~h}$ & 33,9 & 17,0 & 10,2 & 0 & 0 & $\mathrm{nT}$ \\
\hline \multirow[t]{2}{*}{ MA92 } & $24 \mathrm{~h}$ & 24,1 & 10,8 & 0 & 4,6 & 0,3 & \\
\hline & $40 \mathrm{~h}$ & 65,0 & 27,9 & 0 & 2,3 & 0 & $\mathrm{~T}$ \\
\hline \multirow[t]{2}{*}{ MBL93 } & $24 \mathrm{~h}$ & 3,1 & 0 & 0 & 0 & 1,1 & \\
\hline & $40 h$ & 9,0 & 0 & 0 & 0 & 0,9 & nT \\
\hline \multirow[t]{2}{*}{$\mathrm{MBC} 91$} & $24 \mathrm{~h}$ & 9,0 & 0 & 0 & 0 & 0 & \\
\hline & $40 \mathrm{~h}$ & 55,7 & 13,5 & 0 & 0 & 0 & $T$ \\
\hline \multirow[t]{2}{*}{ MG91 } & $24 \mathrm{~h}$ & 93,4 & 78,6 & 60,0 & 11,7 & 6,9 & \\
\hline & $40 \mathrm{~h}$ & 85,8 & 85,8 & 80,0 & 35,1 & 6,9 & $T$ \\
\hline \multirow[t]{2}{*}{ MGL } & $24 \mathrm{~h}$ & 94,9 & 85,1 & 38,5 & 21,2 & 4,5 & \\
\hline & $40 h$ & 88,4 & 88,4 & 85,6 & 68,8 & 31,6 & $\mathbf{T}$ \\
\hline \multirow[t]{2}{*}{ MGR91 } & $24 \mathrm{~h}$ & 0 & 0,6 & 4,0 & 1,9 & 1,9 & \\
\hline & $40 \mathrm{~h}$ & 13,1 & 4,8 & 6,0 & 4,8 & 6,0 & $\mathrm{nT}$ \\
\hline \multirow[t]{2}{*}{ MMC91 } & $24 \mathrm{~h}$ & 14,1 & 5,4 & 0 & 0 & 1,9 & \\
\hline & $40 \mathrm{~h}$ & 36,9 & 8,6 & 1,8 & 2,8 & 0 & $T$ \\
\hline \multirow[t]{2}{*}{ MPM91 } & $24 \mathrm{~h}$ & 1,6 & 0 & 0 & 0 & 0 & \\
\hline & $40 \mathrm{~h}$ & 2,1 & 1,7 & 0,9 & 0 & 0 & nT \\
\hline \multirow[t]{2}{*}{ MU921 } & $24 \mathrm{~h}$ & 7,1 & 0 & 0 & 0 & 6,3 & \\
\hline & $40 \mathrm{~h}$ & 53,2 & 17 & 0 & 0 & 8,1 & $n T$ \\
\hline \multicolumn{8}{|c|}{ Ap. flos-aquae } \\
\hline \multirow[t]{2}{*}{ CCAP1450/4 } & $24 \mathrm{~h}$ & 8,6 & $2 ; 1$ & 0 & 3,8 & 1,7 & \\
\hline & $40 \mathrm{~h}$ & 6,5 & 0 & 4,2 & 4,2 & 3,4 & nT \\
\hline \multirow[t]{2}{*}{ ApG91 } & $24 \mathrm{~h}$ & 8,9 & 7,2 & 5,4 & 6,5 & 0,8 & \\
\hline & $40 h$ & 18,5 & 13,6 & 12,6 & 6,0 & 3,7 & $\mathrm{nT}$ \\
\hline \multirow[t]{2}{*}{ ApI9I } & $24 \mathrm{~h}$ & 16,4 & 8,8 & 8,8 & 4,3 & 2,6 & \\
\hline & $40 \mathrm{~h}$ & 23,5 & 11,9 & 1,6 & 2,2 & 0 & nT \\
\hline \multirow[t]{2}{*}{ ApPM91 } & $24 \mathrm{~h}$ & 0 & 0 & 0 & 0 & 0 & \\
\hline & $40 \mathrm{~h}$ & 16,2 & 4,6 & 0 & 0 & 0 & nT \\
\hline \multirow[t]{2}{*}{ ApR93 } & $24 \mathrm{~h}$ & 0,6 & 1,1 & 0 & 2,4 & 0 & \\
\hline & $40 \mathrm{~h}$ & 17,4 & 8,0 . & 0 & 4,9 & 0 & $\mathrm{nT}$ \\
\hline \multicolumn{8}{|c|}{ An. circinalis } \\
\hline AnGL91 & $24 \mathrm{~h}$ & 88,8 & 67,6 & 20,9 & 3,5 & 4,4 & \\
\hline \multicolumn{8}{|c|}{ Oscillatoria sp. } \\
\hline \multirow[t]{2}{*}{ OBT91 } & $24 \mathrm{~h}$ & 0 & 0 & 0 & 0 & 0 & \\
\hline & $40 h$ & 0 & 0 & 0 & 0 & 0 & $\mathrm{nT}$ \\
\hline \multirow[t]{2}{*}{ OF93 } & $24 \mathrm{~h}$ & 2,5 & 4,0 & 15,1 & 11,6 & 1,3 & \\
\hline & $40 \mathrm{~h}$ & 4,2 & 2,5 & 10,4 & 11,8 & 0 & nT \\
\hline \multirow[t]{2}{*}{ OGL91 } & $24 \mathrm{~h}$ & 3,3 & 0 & 2,9 & 3,1 & 1,3 & \\
\hline & $40 \mathrm{~h}$ & 0,8 & 0 & 0,2 & 0 & 0 & $\mathrm{nT}$ \\
\hline OGR9I & $24 \mathrm{~h}$ & 0 & 0 & 0 & 0 & 0 . & \\
\hline & $40 \mathrm{~h}$ & 0 & 0 & 0 & 0 & 0 & nT \\
\hline
\end{tabular}

Les résultats du test sur Artemia salina sont reportés dans le Tableau 3. Les valeurs indiquées correspondent, pour chaque échantillon, à la moyenne des taux de mortalité des trois essais, après retrait des taux de mortalité moyens obtenus dans les six témoins correspondants. Deux comptages ont été effectués à des 
temps différents pour chaque souche car la mortalité des larves, causée par certaines souches considérées comme hépatotoxiques lors des tests souris, est très faible après 24 heures. En effet, contrairement aux souches MG91, MGL91 et AnGL91 qui provoquent des taux de mortalité supérieurs à $85 \%$ après 24 heures pour la concentration de $10 \mathrm{mg} / \mathrm{ml}$, les souches MA92, MBC91 et MU921 présentent des taux de mortalité très faibles après 24 heures et seulement entre 50 et 65 $\%$ pour cette concentration après 40 heures. Afin d'appliquer la méthode de Kiviranta et al. (1991), seuls les taux de mortalité après 40 heures sont considérés pour déterminer la toxicité des souches. Les souches de $M$. aeruginosa MA92, MG91, MGL91 et d'A. circinalis AnGL91 sont toxiques selon les critères de Kiviranta et al. (1991). Par contre les souches de $M$. aeruginosa MBC91 et MU921 entraînent une mortalité supérieure à $50 \%$ pour la concentration de $10 \mathrm{mg} / \mathrm{ml}$ et inférieure $20 \%$ pour celle de $5 \mathrm{mg} / \mathrm{ml}$.

Les souches non hépatotoxiques pour les souris provoquent une mortalité inférieure à $40 \%$ dans les puits contenant les plus fortes concentrations d'extrait cyanobactérien au bout de 40 heures. La souche MBL93 n'est pas toxique pour les larves d'A. salina et la non toxicité des souches ApPM91 et ApR93 est confirmée.

Les difficultés d'extraction des toxines de certaines souches peuvent expliquer le temps de réaction plus long des larves. L'efficacité d'extraction est liée à la dispersion des cellules däns le mileu. En effet, les souches de $M$. aeruginosa toxiques qui ne donnent pas de résultat positif 24 heures après le début du test (MA92, MBC91 et MU921) se développent sous forme de colonies en milieu liquide. Leurs cellules sont entourées d'une sorte de gel qui permet la formation des colonies (Nakagawa et al. 1987) et rend difficile la lyse des cellules. Par contre, les autres souches de $M$. aeruginosa toxiques (MG91 et MGL91) ont une répartition homogène dans le milieu.

En parallèle à la méthode de Kiviranta et al. (1991), un test de comparaison de moyennes Mann-Whitney a été appliqué aux taux de mortalité. Les valeurs obtenues pour les concentrations de $10 \mathrm{mg} / \mathrm{ml}$ et de 5 $\mathrm{mg} / \mathrm{ml}$ d'un même échantillon ont été regroupées pour les comparer aux valeurs des témoins. Si les différences sont significatives à un seuil de 0.01 après 40 heures de test, les souches sont considérées comme toxiques. Les résultats de ce test sont reportés dans le Tableau 4. D'après celui-ci les souches de $M$. aeruginosa MA92, MG91, MGL91, MU921 et d'A. circinalis AnGL91 sont toxiques. Par contre, la souche de $M$. aeruginosa MBC91 n'est pas toxique alors que la souche de $M$. aeruginosa CCAP1450/1 l'est.
Un test non paramétrique de signe a été appliqué pour comparer la méthode de Kiviranta et al. (1991) ainsi que les résultats du test Mann-Whitney aux résultats d'hépatotoxicité du test souris. Chacune de ces deux méthodes donne deux résultats sur vingt qui ne correspondent pas au test souris. Malgré cela, dans les deux cas, le test de signe ayant donné une valeur de 0.479 , aucune différence significative avec le test souris n'a été montrée.

\section{Conclusion}

Parmi les dix-huit souches provenant du milieu naturel, six ont révélé une hépatotoxicité par les bioessais sur souris ; il s'agit de cinq souches de Microcystis aeruginosa et une souche d'Anabaena circinalis. Une bonne conformité entre le test souris et le test sur Artemia salina a été mise en évidence. L'utilisation des larves de cette crevette marine est donc une méthode efficace pour une pré-détection des hépatotoxines d'autant qu'elle nécessite peu de matériel algal. Manifestement les réservoirs bretons utilisés pour l'alimentation en eau potable sont soumis aux efflorescences

Tableau 4. Résultats du test Mann-Whitney.

* Coefficient calculé à partir des données obtenues après 40 heures de test ( $\mathrm{T}$ toxique ; $\mathrm{nT}$ non toxique).

Table 4. Results of the Mann-Whitney test.

* Coefficient calculated from data obtained following 40 hours test period ( $T$ : toxic, $n T$ : non toxic).

\begin{tabular}{lcc}
\hline \multicolumn{1}{c}{ Souches } & $\begin{array}{c}\text { Coeff.* } \\
\text { Mann-Whitney }\end{array}$ & $\begin{array}{c}\text { Toxicité } \\
\text { (seuil 0,1\%) }\end{array}$ \\
\hline M. aeruginosa & & \\
CCAP1450/1 & 0,005 & $\mathrm{~T}$ \\
MA92 & 0,005 & $\mathrm{~T}$ \\
MBL93 & 1,000 & $\mathrm{nT}$ \\
MBC91 & 0,025 & $\mathrm{nT}$ \\
MG91 & 0,003 & $\mathrm{~T}$ \\
MGL91 & 0,003 & $\mathrm{~T}$ \\
MGR91 & 0,148 & $\mathrm{nT}$ \\
MMC91 & 0,013 & $\mathrm{nT}$ \\
MPM91 & 0,748 & $\mathrm{nT}$ \\
MU921 & 0,005 & $\mathrm{~T}$ \\
Ap. flos-aquae & & \\
CCAP1401/4 & 0,575 & $\mathrm{nT}$ \\
ApG91 & 0,024 & $\mathrm{nT}$ \\
Ap191 & 0,016 & $\mathrm{nT}$ \\
ApPM91 & 0,230 & $\mathrm{nT}$ \\
ApR93 & 0,037 & $\mathrm{nT}$ \\
An. circinalis & & \\
AnGL91 & 0,004 & $\mathrm{~T}$ \\
Oscillatoria sp. & & \\
OBT91 & 1,000 & $\mathrm{nT}$ \\
OF93 & 0,228 & $\mathrm{nT}$ \\
OGL91 & 0,575 & $\mathrm{nT}$ \\
OGR91 & 1,000 & \\
\hline & & \\
\hline
\end{tabular}


de cyanobactéries productrices de toxines. Il serait souhaitable qu'ils fassent l'objet d'une surveillance par les services sanitaires à l'instar de ce qui a été mis en place dans les pays anglo-saxons et scandinaves.

\section{Remerciements}

Cette étude a pu être réalisée grâce à un financement du Conseil Régional de Bretagne.

\section{Travaux cités}

Beasley V.R., Dahlem A.M., Cook W.O., Valentine W.M., Lovell R.A., Hooser S.B., Harada K., Susuki M. \& Carmichael W.W. 1989. - Diagnostic and clinically important aspects of cyanobacterial (blue-green algae) toxicoses. $J$. Vet. Diagn. Invest., 1 : 359-365.

Bourke A.T.C. \& Hawes A.M. 1983. - Freshwater cyanobacteria (blue-green algae) and human health. Med. J. Aust., $28: 491-492$.

Carmichael W.W. \& Falconer I.R. 1993. - Diseases related to freshwater blue-green algal toxins, and control measures. Algal Toxins in Seafood and Drinking Water. Chap., $12: 187-209$.

Carmichael W.W. \& Gorham P.R. 1978. - Anatoxins from clones of Anabaena flos aquae isolated from lakes of western Canada. Mitt. Internat. Verein. Limnol., $21: 285-295$.

Codd G.A. \& Poon G.K. 1988. - Cyanobacterial toxins. In L.J. Rogers and Gallon J.R. (ed). Biochemistry of the algae and Cyanobacteria. Proceeding of the phytochemistry Society of Europe, Oxford University Press, $28: 283-296$.

Falconer I.R., Beresford A.M. \& Runnegar M.T.C. 1983. - Evidence of liver damage by toxin from a bloom of the blue-green alga, Microcystis aeruginosa. Med. J. Aust., 1 ; 511-514.

Hughes E.O., Gorham P.R. \& Zehnder A. 1958. - Toxicity of a unialgal of Microcystis aeruginosa. Can. J. Microbiol., $4: 225$. 037
Kiviranta J., Sivonen K., Niemala S.I. \& Huovinen K. 1991. - Detection of toxicity of Cyanobacteria by Artemia salina Bioassay. Environ. Toxic. Wat. Qual., 6 (4) : 423-436.

Luukkainen R., Sivonen K., Namikoshi M., Fardig M., Rinehart K.L. \& Niemala S.I. 1993. - Isolation and identification of eight microcystins from thirteen Oscillatoria agardhii strains and structure of a new microcystin. Appl. Environ. Microbiol., 59 (7) : 22042209.

Nakagawa M., Takamura Y. \& Yago. 1987. - Isolation and characterization of the slime from a cyanobacterium, Microcystis aeruginosa K-3A. Agric. Biol. Chem., 51 (2) : 329-337.

Shirai M., Matumaru K., Ohotake A., Takamura Y., Aida T. \& Nakano M. 1989. - Development of a solid medium for growth and isolation of axenic Microcystis strains (Cyanobacteria). Appl. Environ. Microbiol.. 55 (10) : 2569-2571.

Sivonen K., Niemala S.I., Niemi R.M., Lepisto L., Luoma T.H. \& Rasanel L.A. 1990. - Toxic cyanobacteria (blue-green algae) in finnish fresh and coastal waters. Appl. Environ. Microbiol., 190: 267:275

Skulberg O.M., Cood G.A. \& Carmichael W.W. 1984. - Toxic blue-green blooms in Europe : a growing problem. Ambio., 13 (4) :244-247.

Theiss W.C., Carmichael W.W., Wyman J. \& Bruner R. 1988. Blood pressure and hepatocellular effects of the cyclic heptapeptide toxin produced by the freshwater cyanobacterium (bluegreen alga) Microcystis aeruginosa strain PCC-7820. Toxicon., 26 (7) : 603-13

Watanabe M.F., Watanabe M., Kato T., Harada K-I. \& Susuki M. 1991. - Composition of cyclic peptide toxins among strains of Microcystis aeruginosa (blue-green algae, Cyanobacteria). Bot. Mag., Tokyo, $104: 49-57$. 